\title{
Aggregation Aware Spectrum Assignment in Cognitive Ad-hoc Networks
}

\author{
Dawei Chen \\ The Hong Kong University of \\ Science and Technology \\ Hong Kong \\ dwchen@cse.ust.hk
}

\author{
Qian Zhang \\ The Hong Kong University of \\ Science and Technology \\ Hong Kong \\ qianzh@cse.ust.hk
}

\author{
Weijia Jia \\ City University of Hong Kong \\ Hong Kong \\ wei.jia@cityu.edu.hk
}

\begin{abstract}
Contiguous spectrum assignment generates many small spectrum fragments that cannot be fully utilized, which leads to low spectrum utilization. With the development of advanced wireless radio technology, especially Discontiguous Orthogonal Frequency Division Multiplexing (DOFDM), discontiguous spectrum access and spectrum aggregation in a single radio has become possible. With the help of discontiguous spectrum access, the small spectrum fragments could be aggregated and further utilized, which can dramatically improve the spectrum utilization efficiency. Based on this observation, we propose Aggregation-Aware Spectrum Assignment (AASA), a spectrum assignment algorithm in cognitive ad-hoc networks. In AASA, we propose a spectrum assignment method that takes spectrum aggregation into consideration. Moreover, we prove that AASA optimizes the spectrum assignment and maximizes the number of users that can be supported in the system. Simulation result shows that AASA significantly increases the spectrum assignment efficiency compared with the existing solutions.
\end{abstract}

Index Terms-AASA, Wireless Networks, Cognitive Radio, Spectrum Aggregation, DOFDM

\section{INTRODUCTION}

Cognitive Radio Network (CRN) is becoming more and more popular in recent years. In traditional wireless networks, the lack of radio spectrum has become the bottleneck of the development of wireless networks. On the other hand, the spectrum utilization efficiency in licensed spectrum bands is quite low [4]. Thus, people are exploiting new way to transmit on licensed bands for wireless networks when these bands are not fully used.[1][2][3]

In CRN, one of the challenging problems is how to assign the available spectrum efficiently. Intuitively speaking, assume that each transmission pair demands a certain bandwidth of spectrum for transmitting, and then the problem is how to assign the spectrum to support as many transmission pairs as possible.

Spectrum/channel assignment is a well-studied problem in traditional wireless networks. [5] provides a comprehensive survey in the area of dynamic, fixed and hybrid allocation strategy, and compares their complexity, performance and the trade-offs.

However in CRN, compared to the traditional wireless networks, the fundamental difference is that the available spectrum bands/channels are dynamic and their availability

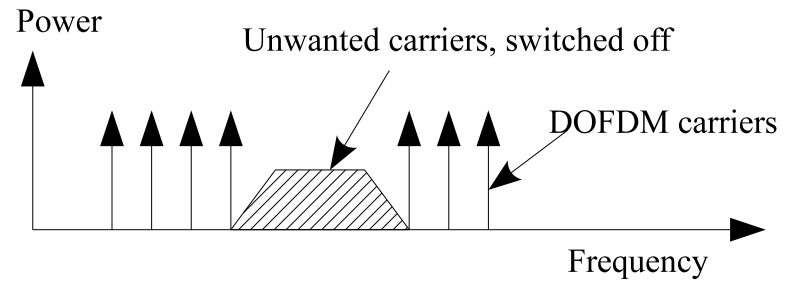

Fig. 1. Spectrum aggregation using DOFDM. DOFDM carriers are on two available spectrum bands and can be aggregated into one channel.

changes all the time, which makes the traditional spectrum/channel assignment protocols do not work. Because of this fundamental difference, new protocols/standards are proposed, such as the ones in [7][8] and the well-known IEEE 802.22[9]. For high level understanding, [6] provides a survey. In [8], a Dynamic Open Spectrum Sharing (DOSS) MAC protocol is proposed: when assigning spectrums for a transmission between two users, they first negotiate on a pre-known control channel to tell each other their own available spectrum bands. In their common available bands, they choose the largest contiguous band, on which they start transmitting. During transmitting, if the transmission band turns unavailable, they suspend current data transmission and re-assign the spectrum. After a new spectrum band is assigned, the data transmission is resumed on the new band. So although the available spectrum bands are dynamic and their availability changes all the time in CRN, DOSS still works.

However the above works are based on contiguous channel assignment algorithms. In current contiguous channel assignment algorithms, each channel consists of only one contiguous spectrum fragment, so they cannot utilize small spectrum fragments whose bandwidths are smaller than the users' demand. Moreover these algorithms generate more fragments, which makes the spectrum utilization efficiency low.

Fortunately with the development of advanced wireless radio technology, especially Discontiguous Orthogonal Frequency Division Multiplexing (DOFDM)[13], a single radio can access several spectrum fragments simultaneously, thus aggregates them into one channel[10]. In Figure I, DOFDM carriers are on two small spectrum fragments. The spectrum 
Available spectrum fragments

Existing services

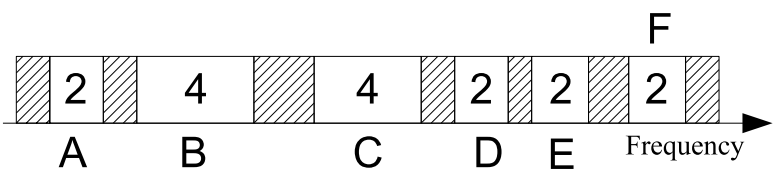

Fig. 2. One example of spectrum fragments. The letters are the labels of the available bands. The numbers are the bandwidths of the bands $(\mathrm{MHz})$

band between the two fragments is occupied by primary users (thus unavailable). By switching off the carriers on the unavailable band, the data could be transmitted simultaneously on the two spectrum fragments by using one single radio.

In Figure I, if the bandwidth of each fragment is smaller than the demand, they will not be utilized by contiguous spectrum assignment. However, by aggregating them into one channel, the aggregated bandwidth might be larger than the demand. In this way, small fragments are fully utilized to improve the spectrum efficiency.

For example, if in one CRN every transmission pair demands $4 \mathrm{MHz}$, while the available bands are four $2 \mathrm{MHz}$ bands and two $4 \mathrm{MHz}$ bands, respectively, as shown in Figure 2. In Figure 2 the white parts are available spectrum bands and the number is the bandwidth $(\mathrm{MHz})$, while the hatching parts are unavailable. With contiguous spectrum assignment, it cannot utilize the four $2 \mathrm{MHz}$ bands because they are smaller than the demand $4 \mathrm{MHz}$. It can only utilize the $4 \mathrm{MHz}$ bands ( $\mathrm{B}$ and C). So this network can only support 2 transmission pairs. In this case, contiguous assignment utilized $8 \mathrm{MHz}$ out of total $16 \mathrm{MHz}$ available spectrum. If we could aggregate small bands into one channel, we could fully utilize $16 \mathrm{MHz}$ and support 4 transmission pairs. In most cases, in one CRN, after spectrum sensing and assignment for a period of time, there are always many small spectrum fragments that cannot be utilized individually unless they are aggregated.

The above example shows the great advantage of spectrum aggregation in spectrum assignment. However, there are also some limitations on spectrum aggregation: for example, the span of the aggregated bands is not unlimited, due to the limitation of the transceivers [11][12]. For a transceiver, if the max span is $10 \mathrm{MHz}$, all the spectrum bands which it is using must be within a $10 \mathrm{MHz}$ band.

With this constraint, some spectrum fragments may not be aggregated because their span is larger than the limited max span.

From above we could see that spectrum aggregation will help greatly to improve the spectrum assignment result. But the aggregation has also limitations, which may make spectrum assignment complicated. So when assigning spectrum, we should avoid generating new fragments that cannot be aggregated. Fortunately we observe that we could achieve this by using a simple greedy algorithm that search for and assign channels from low to high frequency.

Thus motivated by the above description, in this paper we:
1) Propose Aggregation Aware Spectrum Assignment (AASA), a spectrum assignment algorithm. We apply spectrum aggregation in AASA under the limitation that the max span of a channel is limited.

2) Prove theoretically that AASA optimizes the spectrum assignment and maximizes the number of supported users.

3) Study the performance of AASA by simulation.

The simulation result shows that AASA significantly increased the number of supported users in cognitive ad-hoc networks compared to the contiguous spectrum assignment algorithms.

The rest of the paper is organized as follows: Section 2 introduces our motivation and gives the problem definition. Then we proposed AASA to solve the problem in Section 3. We prove in detail that AASA optimizes the spectrum assignment in Section 4. Simulation for AASA is in Section 5. Finally we conclude this paper in Section 6 .

\section{Motivation And Problem Definition}

\section{A. Motivation}

In the introduction section we showed the advantage of spectrum aggregation. However, even with spectrum aggregation, different strategies lead to different results, as demonstrated in the following example.

\section{Motivated Example:}

In Figure 2, assume the max span of a channel is $10 \mathrm{MHz}$. If the spectrum assignment strategy is trying to use the wide bands first, the result might be that it assigns the spectrum to 3 transmission pairs: one with band $\mathrm{B}$, one with $\mathrm{C}$, one with the aggregation of $\mathrm{E}$ and $\mathrm{F}$. However $\mathrm{A}$ and $\mathrm{D}$ cannot be aggregated because their span is larger than $10 \mathrm{MHz}$, so they are left unutilized.

In fact, in this example, the network can support 4 transmission pairs, one with band $\mathrm{A}$ and half $\mathrm{B}$, one with half $\mathrm{B}$ and half $\mathrm{C}$, one with half $\mathrm{C}$ and $\mathrm{D}$, the other one with $\mathrm{E}$ and F.

In this example, we evaluate the performances of the spectrum assignment strategies by their numbers of supported transmission pairs. If there are always transmission requests and every transmission pairs demand the same bandwidth, then the more transmission pairs it supports, the better the spectrum assignment strategy is.

\section{B. Problem definition}

Before stating the problem, we define these notations:

Node: A node is a transmission pair. It could either be transmitting or just request for transmitting but not start.

Channel: In this paper, a channel consists of either one contiguous spectrum band or some discontiguous bands.

Working Node:If a node is transmitting, it is a working node.

\section{Assumptions:}


1) The available spectrum bands are fixed and known as $F=\bigcup_{i=1}^{q}\left[F_{i L}, F_{i U}\right]$ where $\mathrm{q}$ is a known constant, $F_{i L}<F_{i U}, \forall i=1,2 \ldots q$ and $F_{i U}<F_{(i+1) L}, \forall i=$ $1,2 \ldots q-1$

2) There are $K$ nodes in one $C R$ ad-hoc network, labeled from 1 to $\mathrm{K}$.

3) Each node demands a channel with $\mathrm{R} \mathrm{Hz}$ ( $\mathrm{R}$ is a known constant) bandwidth; otherwise the node cannot start transmitting. Here a channel could consist of some discontiguous spectrum bands. But the span of a channel must be smaller than $M A \mathrm{~Hz}$.

4) In this network there is a broker. It determines what spectrum bands a channel consists of, and what channel a node could work on.

5) All the nodes interfere with each other.

6) We consider a saturated system and assume there are always transmission requests.

Let $C_{m}$ indicates Channel $\mathrm{m}$, whose bandwidth is $\left|C_{m}\right| \mathrm{Hz}$. $C_{m}$ consists of $t_{m}$ spectrum fragments.

$$
\begin{aligned}
C_{m} & =\bigcup_{i=1}^{t_{m}}\left[f_{m L}^{i}, f_{m U}^{i}\right] \subseteq F \\
\left|f_{m U}^{i}-f_{m L}^{j}\right| & \leq M A, \quad \forall 1 \leq i, j \leq t_{m} \\
\left|C_{m}\right| & =\sum_{i=1}^{t_{m}}\left|f_{m U}^{i}-f_{m L}^{i}\right| \\
C_{i} \cap C_{j} & =\emptyset, \quad \forall i \neq j
\end{aligned}
$$

$M A$ is the max span of a channel.

Working node $\mathrm{m}$ works on $C_{m}$. Its demand bandwidth is $R$ Hz. So $\left|C_{m}\right| \geq R$.

\section{The problem:}

Let $N$ indicates the number of working nodes. Then the problem is to maximize $N$, subject to

$$
\sum_{m=1}^{N}\left|C_{m}\right| \leq|F|,\left|C_{m}\right| \geq R
$$

\section{AASA: THE PROPOSED ALGORITHM}

To solve the given problem, we propose Aggregation Aware Spectrum Assignment (AASA) algorithm.

Let $\mathrm{N}$ indicates the number of the working nodes. The goal of AASA is to maximize N.

What's the basic idea for AASA? Intuitively speaking, if a network can originally support $\mathrm{n}$ working nodes, the basic idea to optimal the spectrum assignment strategy is to keep the network capable to support $(n-m)$ nodes after assigning spectrum to $m(m<n)$ nodes. In the motivated example in Section 2, the network can originally support 4 nodes. But "wide bands first" strategy makes it only able to support 1 node after assigning band $\mathrm{B}$ and $\mathrm{C}$ to 2 nodes. We call this network capability loss.

The basic idea for AASA is to avoid network capability loss.

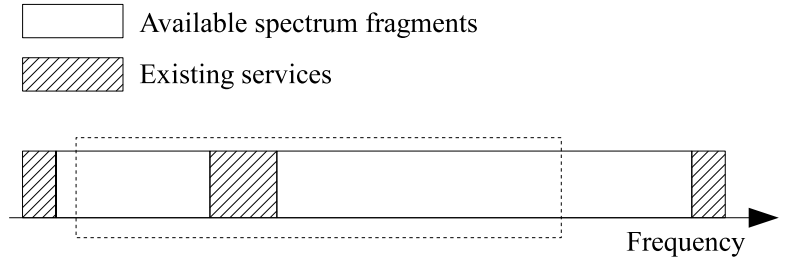

Fig. 3. Spectrum availability. The width of the dash-line rectangle indicates the max span of a channel.

AASA is a simple greedy algorithm: it searches for the channels from left to right (from low to high frequency). Once it finds out a channel that the bandwidth is $R \mathrm{~Hz}$, AASA assigns it to a node.

The availability of the spectrum could be shown as Figure 3 . The width of the dash-line rectangle in Figure 3 indicates $M A$, the max span of a channel.

AASA is explained based on Figure 3.

1) Initially locate the dash line rectangle at the left edge of the whole spectrum. Let $v=0, S=\emptyset$

2) If the window moves out of the right edge, go to 7 .

3) Is there available $R \mathrm{~Hz}$ in this rectangle? If yes, go to 4. Otherwise go to 5 .

4) Aggregate the most left available $R \mathrm{~Hz}$ to one channel and assign it to a node. Then mark the corresponding spectrum unavailable. $v \leftarrow v+1$. Let $S_{v}$ indicates the frequency set of this channel, $t_{v}$ indicates the number of fragments of this channel. Then

$$
S_{v}=\bigcup_{i=1}^{t_{v}}\left[f_{v L}^{i}, f_{v U}^{i}\right], \quad S_{v} \subseteq F
$$

5) Move the dash line rectangle to the right.

6) Goto 2

7) Let $S=\left\{S_{i} \mid i=1,2 . . v\right\}$. Stop

After running the algorithm, $S$ is the result channel set and $v$ is the number of working nodes. $S_{i}$ is the $i$ th channel in $\mathrm{S}$ and it consists of $t_{i}$ spectrum bands.

$$
\begin{aligned}
S & =\left\{S_{i} \mid i=1,2 . . v\right\} \subseteq F \\
S_{i} & =\bigcup_{k=1}^{t_{i}}\left[f_{i L}^{k}, f_{i U}^{k}\right]
\end{aligned}
$$

This $\mathrm{S}$ has these properties:

1) $\left|S_{i}\right|=R, S_{i} \cap S_{j}=\emptyset, \forall i \neq j$

2) $\forall 1 \leq i<j \leq v, x \in S_{i}, y \in S_{j}$, we have $x<y$

3) For a channel $S_{i}$, the frequency between $f_{i L}^{1}$ and $f_{i U}^{t_{i}}$ is fully utilized. That is:

$$
\left[f_{i L}^{1}, f_{i U}^{t_{i}}\right] \cap F=S_{i}
$$

4) AASA aggregates the most left available $R \mathrm{~Hz}$. That is:

$\forall k, i \quad$ s.t. $\quad k<f_{(i+1) L}^{1}$

Let $A=[k, k+M A], B=S_{1} \cup S_{2} \ldots \cup S_{i}$

Then $|(A-B) \cap F|<R$

5) AASA cannot find any more channels. That is

$$
|[k, k+M A] \cap F|<R, \forall k \geq f_{v U}^{t_{v}}
$$




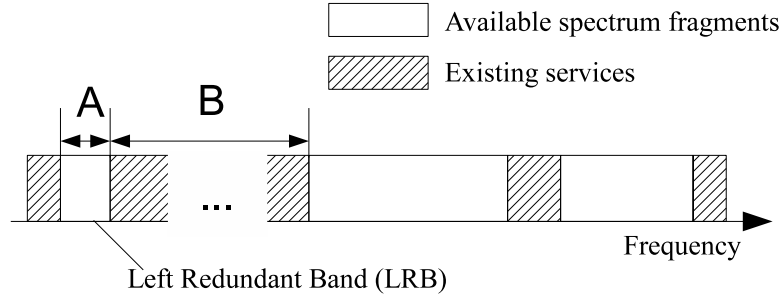

Fig. 4. An example of the left redundant band. A is the most left available fragment in this network and its bandwidth is smaller than the demand. B is wider than the span of a channel. A cannot be aggregated with other fragments because they are too far away.

Because of Property 4, Property 5 is equivalent to: $\forall k$, let $A=[k, k+M A]$, then $|(A-S) \cap F|<R$ $M A$ is the max span of a channel.

AASA only scan the spectrum once. The time complexity of AASA is $\mathrm{O}(\mathrm{M})$ where $\mathrm{M}$ is the span of the whole spectrum.

\section{ProOF OF AASA}

In this section we prove that AASA maximizes the number of supported working nodes. In other words, we prove that if a network can support maximum $N$ nodes, then AASA will find $N$ channels and assign them to $N$ nodes in this network.

We use induction method and the three steps are:

1) If a network can only support one working node, we prove that AASA will find one channel with demand bandwidth.

2) Assume that if a network can support $k$ working nodes, AASA will find $k$ channels. We prove that if a network can support $k+1$ working nodes, AASA will find $k+1$ channels with demand bandwidth.

3) If the above 2 steps are done, we draw the conclusion that AASA maximizes the number of supported working nodes.

In order to keep the proof simple and brief, we first discard some "noise" in the spectrum bands. Here "noise", we mean the apparently unusable spectrum fragments. Suppose there is a small available spectrum fragments too far away from any other available fragments that it cannot be aggregated into a channel. Then we could just simply ignore this fragment because it cannot be utilized in any ways. Here we introduce Theorem 1:

THEOREM 1: in a network, the available spectrum bands are $B_{1}, B_{2} \ldots B_{n} . B_{i}$ is sorted and $B_{1}$ is the most left (frequency is the lowest) band. The demand bandwidth of a node is $R \mathrm{~Hz} .\left|B_{1}\right|<R$ and $B_{1}$ cannot be aggregated with other bands into a channel with the demand bandwidth. Then after discarding $B_{1}$ this network supports the same number of nodes as before discarding. $B_{1}$ is called the left redundant band (LRB).

Theorem 1 is illustrated with Figure 4. It is obvious and we do not prove it in this paper.
In dynamic environment, spectrum availability changes all the time so actually $B_{1}$ in Theorem 1 might be aggregated with some new available spectrum bands in the future. But in this paper we only consider the static spectrum availability.

In a network, we may find many kinds of spectrum bands that cannot be utilized. But for the proof of AASA, it is just enough to find LRBs described in Theorem 1.

Now we start our proof of AASA:

\section{Induction step 1:}

If a network can only support one node, we prove that AASA will find one channel with demand bandwidth.

In fact, AASA scans the whole spectrum in this network. So if there is a channel, AASA will absolutely find it.

\section{Induction step 2:}

Assume that if a network can support $k$ nodes, AASA will find $k$ channels. We need to prove that if a network can support $k+1$ working nodes, AASA will find $k+1$ channels with demand bandwidth.

Our thread for proof in this step is: first divide the network spectrum into two parts: one part supports only one working node and the other supports $k$ working nodes. However it is possible that after dividing, one part of spectrum supports only one working but the other part cannot support $k$ working nodes, if the original network spectrum is not carefully divided.

So the difficulty is how to divide the spectrum. To simplify the problem, we only consider the network that has no LRBs, because according to Theorem 1 if a network has LRBs, simply discard the LRBs and the number of supported working nodes does not change. For the spectrum dividing, we introduce Theorem 2:

THEOREM 2: A working node demands a channel with $R \mathrm{~Hz}$ bandwidth. A network has no LRBs and can support $n$ working nodes. Then if we discard available $R \mathrm{~Hz}$ from the most left of the available spectrum, then the rest of the spectrum can support $n-1$ working nodes.

We left the proof of Theorem 2 in the appendix to keep this section brief.

We first discard all the LRBs and then divide the spectrum into two parts: the first part is the most left $R \mathrm{~Hz}$ available spectrum, the second part is the rest. Obviously the first part can support one working node. According to Theorem 2, the second part can support $\mathrm{n}$ working nodes.

Apparently, AASA will find the whole first part as the first channel, and then it continues its channel searching in the second part. According to the assumption in Induction Step 2, AASA will find $k$ channels in the second part.

So if a network can support $k+1$ working nodes, AASA will find $k+1$ channels with demand bandwidth.

\section{Induction step 3:}


TABLE I

THE CONFIGURATIONS OF THE SIMULATION.

\begin{tabular}{l||l}
\hline Frequency range & $100 \mathrm{MHz}-700 \mathrm{MHz}$ \\
Total available bandwidth & $300 \mathrm{MHz}$ \\
Total unavailable bandwidth & $300 \mathrm{MHz}$ \\
Demand bandwidth per node & $10 \mathrm{MHz}$ \\
Max span of a channel & $40 \mathrm{MHz}$ \\
\hline
\end{tabular}

From Induction Step 1 and Step 2, we draw the conclusion that for any $n$, if a network can support $n$ working nodes, AASA will find $n$ channels with demand bandwidth.

So we finished the proof and draw the conclusion that AASA maximizes the number of supported working nodes.

\section{Simulation And Analysis}

In this section, we evaluate the performance of AASA by computer simulation and compare the performance to the contiguous assignment algorithm.

The methodology we follow in our simulation is to isolate the impact of the total bandwidth of the available bands and the whole span of the spectrum. We only study the effect of the number of the fragments. Intuitively, more fragments there are, more scattered the available bands are.

To isolate the impact of the total bandwidth of the available bands and the whole span of the spectrum, our simulation is taken on a fixed frequency band from $100 \mathrm{MHz}$ to $700 \mathrm{MHz}$ (within broadcasting TV band) and the total bandwidth of all the available bands is fixed to $300 \mathrm{MHz}$. The max span of a channel is mainly imposed by the sampling speeds of today's analogue-to-digital converters and they are usually larger than $40 \mathrm{MHz}$ [12]. So we choose $40 \mathrm{MHz}$ as the max span of a channel. The demand bandwidth per node is set to $10 \mathrm{MHz}$, as it satisfies most of the applications.

In summary, the configurations are shown in Table I.

We only study the effect of the number of the fragments. So we change the number of the fragments from 1 to 100. In every number of fragments, we run AASA and the traditional spectrum assignment algorithm for 100 times respectively and get their average number of supported working nodes. In every run, the spectrum availability is re-generated randomly.

The simulation result is shown in Figure 5.

From Figure 5 we could see that when the fragments number is 1 (i.e. there is only one contiguous spectrum band), the performances are the same. But as the number of fragments increases, the supported number of working nodes of contiguous spectrum assignment become less and less. Intuitively speaking, this is because as the number of fragments increases, the available spectrum bands become more and more scattered, which makes the contiguous assignment algorithm not suit. On the other hand, the number of fragments does not affect AASA much and AASA always get very high number of working nodes. Please note that the total available bandwidth is $300 \mathrm{MHz}$ and the demand bandwidth per node is $10 \mathrm{MHz}$, so the max number of supported working node is 30 in theory in

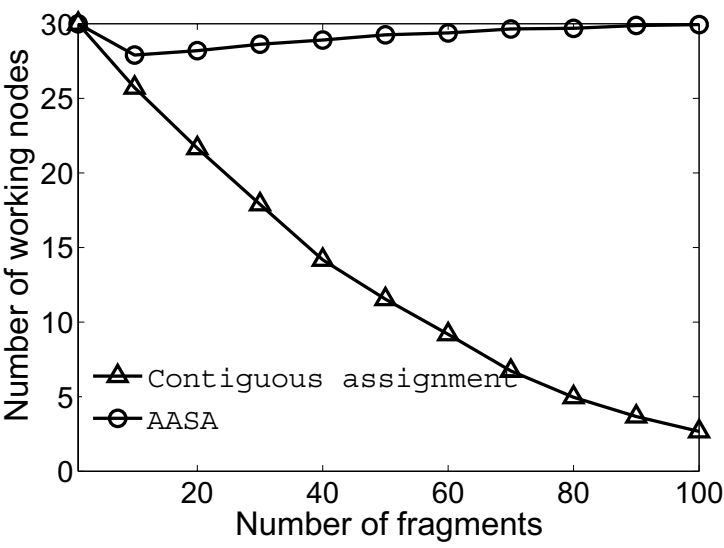

Fig. 5. Number of supported working nodes of contiguous spectrum assignment and AASA.

any cases. The supported number of working nodes of AASA is very close to this theoretical max value in this simulation.

\section{CONCLUSION}

In this paper, we proposed AASA, a spectrum assignment algorithm in cognitive ad-hoc networks. Through aggregating small spectrum fragments to one channel, AASA utilizes the small spectrum fragments that cannot be utilized by contiguous spectrum assignment algorithms. This makes the spectrum utilization efficiency of AASA higher than contiguous spectrum assignment. The main contributions of our paper are:

1) Propose Aggregation Aware Spectrum Assignment (AASA), a spectrum assignment algorithm. The complexity of AASA is $O(M)$ where $M$ is the span of the whole spectrum.

2) Prove theoretically that AASA optimizes the spectrum assignment and maximizes the number of supported users.

3) Evaluate the performance of AASA by simulation. Simulation demonstrates that AASA outperforms the contiguous spectrum assignment schemes significantly.

One of the drawbacks of AASA is that it assumes the spectrum availability does not change, which is not true in the real network environments. We will leave this to our future work.

\section{APPENDIX \\ PROOF OF THEOREM 2}

THEOREM 2: A working node demands a channel with a bandwidth of $R \mathrm{~Hz}$. a network has no LRBs and supports $n$ working nodes. Then if we discard available $R \mathrm{~Hz}$ from the most left of the available spectrum, then the rest of the spectrum supports $n-1$ working nodes.

Please note that the network is based on our assumptions in the "Motivation and Problem Definition" section.

To prove it, we build $n$ channels from this network. Then we adjust one channel (Channel A) to the most left $R \mathrm{~Hz}$. 
Available spectrum fragments,

not assigned to any channels

A Available spectrum fragments, assigned to Channel $\mathrm{A}$

Existing services

\begin{tabular}{|l|l|l|l|l|l|l|l|}
\hline & A & B $/ / A$ & B & C & B & C \\
Frequency
\end{tabular}

Fig. 6. An example of spectrum assignment. Spectrum is assigned to Channel A, B, C. The bandwidth of a channel is $\mathrm{R} \mathrm{Hz}$

Available spectrum fragments, not assigned to any channels

A Available spectrum fragments, assigned to Channel A

Existing services

\begin{tabular}{|l|l|l|l|l|l|}
\hline & A & B & B & C & B \\
Frequency
\end{tabular}

Fig. 7. After exchanging, Fig.6 becomes like this. All the spectrum fragments of Channel A are at the left of any other channels.

After that, "discard $R \mathrm{~Hz}$ from the most left of the available spectrum" in Theorem 2 is "discard Channel A" In this case, it is obvious that the rest of the spectrum supports $n-1$ working nodes, because there are $n-1$ channels in the rest of the spectrum.

\section{PROOF:}

This network support $n$ working nodes. Therefore there are $n$ non-overlapping channels. For example, Figure 6 shows that spectrum assign to 3 channels. The most left spectrum band is not assign to any channels.

The most left occupied spectrum is belong to Channel A. So compare and exchange the spectrum bands between Channel A and all the other channels to make the spectrum fragments of Channel A at the left of any other channels. After exchanging, Figure 6 will become Figure 7 .

Then we move Channel A to the most left side of the spectrum. So the most left available $R \mathrm{~Hz}$ spectrum is occupied by Channel A, showed in Figure 8 .

If we discard Channel $\mathrm{A}$, there are 2 channels in the rest part. So if we discard $R \mathrm{~Hz}$ from the most left of the available spectrum, the rest of the spectrum supports 2 working nodes.

Although we explained the proof by this particular example, the spectrum exchanging and moving can be applied to any networks. And we conclude that if we discard $R \mathrm{~Hz}$ from the most left of the available spectrum, the rest of the spectrum supports $n-1$ working nodes.
Available spectrum fragments, not assigned to any channels

Available spectrum fragments, assigned to Channel $\mathrm{A}$

Existing services

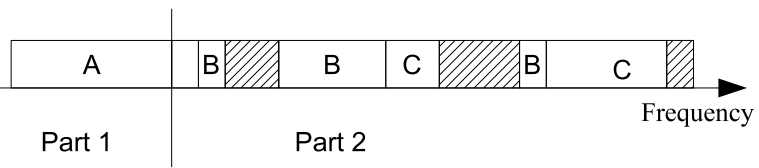

Fig. 8. Move Channel A in Figure 7 to the left. On the right edge of Channel $\mathrm{A}$, the spectrum is divided into two parts; the left is part 1 and the right is part 2.

\section{ACKNOWLEDGEMENT}

The research was support in part by grants from RGC under the contracts CERG 622407 and N_HKUST609/07, by grant from HKUST under the contract RPC06/07.EG05, by grant from National Basic Research Program of China (973 Program) under the contract No. 2006CB303100, the NSFC oversea Young Investigator grant under grant no. 60629203, National 863 Program of China under Grant No. 2006AA01Z228.

\section{REFERENCES}

[1] J. Mitola et al. "ognitive Radios: Making Software Radios more Personal", IEEEE Personal Communications, vol.6, no.4, August 1999.

[2] J. Mitola. "Cognitive radio: An integrated agent architecture for software defined radio". PhD Dissertation, Royal Inst. Technol. (KTH), Stockholm, Sweden, 2000.

[3] S. Haykin. "Cognitive Radio: Brain-Empowered Wireless Communication" in IEEE JSAC, vol23, no.2, February 2005.

[4] FCC, ET Docket No 03-222 Notice of proposed rule making and order, December 2003

[5] I. Katzela, M. Naghshineh. "Channel assignment schemes for cellular mobile telecommunicationsystems: a comprehensive survey". Personal Communications, IEEE June 1996

[6] IF Akyildiz, WY Lee, MC Vuran and S Mohanty. "NeXt generation/dynamic spectrum access/cognitive radio wireless networks: A survey". Computer Networks, May 2006

[7] Qing Zhao, Lang Tong, and Ananthram Swami. "DECENTRALIZED COGNITIVE MAC FOR DYNAMIC SPECTRUM ACCESS”. DySPAN 2005

[8] Liangping Ma, Xiaofeng Han, Chien-Chung Shen. "Dynamic open spectrum sharing MAC protocol for wireless ad hoc networks". DySPAN 2005

[9] C. Cordeiro, K. Challapali, D. Birru and N. Sai Shankar. "IEEE 802.22: the first worldwide wireless standard based on cognitive radios". DySPAN 2005

[10] A.M. Wyglinski. "Effects of Bit Allocation on Non-contiguous Multicarrier-based Cognitive Radio Transceivers". VTC 2006

[11] Qian Zhang, Juncheng Jia and Xuemin Sherman Shen, chapter "Optimal Spectrum Sensing Decision for Hardware-Constrained Cognitive Networks" in book "ognitive Wireless Communication Networks". Springer US, 2007

[12] A. Shukla, B. Willamson, J. Burns, E. Burbidge, A. Taylor, and D. Robinson, "A study for the provision of aggregation of frequency to provide wider bandwidth services". A report. 2006

[13] J. D. Poston and W. D. Horne, "Discontiguous OFDM considerations for dynamic spectrum access in idle TV channels" in Proc. IEEE Int. Symp. New Frontiers Dynamic Spectr. Access Networks, Baltimore, MD, Nov. 2005 , vol. 1 , pp. 607-610. 